\title{
Trivium
}

Revue franco-allemande de sciences humaines et sociales - Deutsch-französische Zeitschrift für Geistesund Sozialwissenschaften

$31 \mid 2020$

La culture politique de la République romaine

\section{Die domus, Instrument des aristokratischen} Prestiges

\section{Jean-Pierre Guilhembet}

Traducteur : Andreas Wittenburg

\section{CpenEdition}

\section{Journals}

Édition électronique

URL : https://journals.openedition.org/trivium/7201

DOI : 10.4000/trivium.7201

ISBN : 1963-1820

ISSN : 1963-1820

\section{Éditeur}

Les éditions de la Maison des sciences de l'Homme

\section{Référence électronique}

Jean-Pierre Guilhembet, „Die domus, Instrument des aristokratischen Prestiges“, Trivium [Online], 31 | 2020, online erschienen am 30 Juni 2020, abgerufen am 24 August 2021. URL: http:// journals.openedition.org/trivium/7201 ; DOI: https://doi.org/10.4000/trivium.7201

Ce document a été généré automatiquement le 24 août 2021.

\section{(c) $(1)$}

Les contenus des la revue Trivium sont mis à disposition selon les termes de la Licence Creative Commons Attribution - Pas d'Utilisation Commerciale - Pas de Modification 4.0 International. 


\title{
Die domus, Instrument des aristokratischen Prestiges
}

\author{
Jean-Pierre Guilhembet
}

Traduction : Andreas Wittenburg

\section{NOTE DE L'ÉDITEUR}

\section{Originalausgabe | édition originale}

"La domus, instrument de prestige aristocratique«, in: Baudry, R. / Hurlet, Fr. (Hg.) : Le Prestige à Rome à la fin de la République et au début du Principat, Paris, De Boccard, 2016, S. 179-191.

Wir danken Herrn Jean-Pierre Guilhembet und dem Verlag De Boccard für die freundliche Genehmigung, diesen Artikel in deutscher Übersetzung zu publizieren. Nous remercions M. Jean-Pierre Guilhembet et les Éditions de Boccard de nous avoir accordé l'autorisation de traduire ce texte pour le présent numéro.

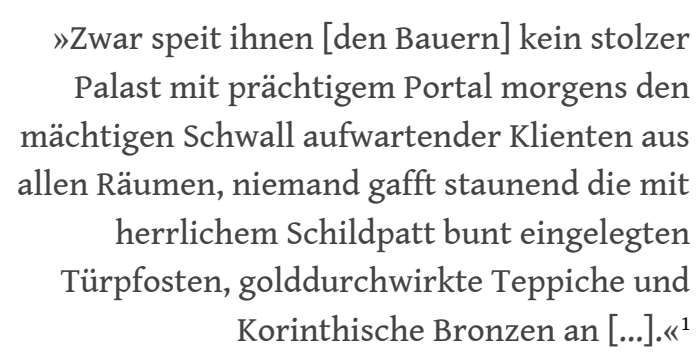

Unter den Strategien der öffentlichen Repräsentation und den wirkungsvollen Verhaltensweisen, deren sich die römische Aristokratie der Republik bediente, geht die Zurschaustellung und Instrumentalisierung des städtischen Wohnsitzes nach der Überlieferung mindestens bis auf den Sturz der Monarchie zurück, wenn nicht auf den eigentlichen Beginn der Stadt. ${ }^{2}$ In dem Zeitraum, um den es hier geht, d. h. die letzten Jahrzehnte der Römischen Republik und die augusteische Epoche oder, genauer gesagt, die Zeit zwischen Pompeius und Augustus, ${ }^{3}$ reiht sich die domus ganz und gar in die Szenografie des politischen Theaters ein. Sie ist Ort und Rahmen der Handlungen und 
Verhaltensweisen, die das Prestige der Aristokratie hervorheben, aber sie kann auch an und für sich als ein Instrument des Prestiges verstanden werden. Sie ist von der Stadt nicht mit einem besonderen Status bedacht, denn nach unserer Kenntnis erwähnt kein spezielles Reglement ihren Besitz, ${ }^{4}$ doch sie trägt durch verschiedene wesentliche Begriffe zum Prestige bei: Anziehungskraft, Bewunderung, Respekt, Auszeichnung. Dieser Gesichtspunkt, den man mit gutem Recht funktionalistisch nennen könnte, führt dazu, dass das ideale Stadthaus von Autoren wie Cicero oder Vitruv "theoretisch" und unter dem Aspekt der dignitas gesehen wird. Ersterer fasst sie als hominis honorati et principis domus ins Auge, Letzterer als die Residenz eines Mannes, der honores magistratusque innehat, selbst wenn er nicht zögert, bei der Formulierung seiner Überlegungen ein so bedeutungsschweres Wort wie regalis zu verwenden. ${ }^{5}$ Doch im vorliegenden Zusammenhang wird sich die Untersuchung häufiger auf den Gesichtspunkt des Nutzens richten, da das Stadthaus durch mehrere Verwendungen unterschiedlicher Art erlaubt, Prestige zur Schau zu tragen, aber auch anzusammeln.

Diesen Ansatz kann man verdeutlichen, indem man sich zunächst auf einen besonderen historischen Zeitpunkt, auf eine wahrhaft beispielhafte und bezeichnende Phase der Spannungen und der Konkurrenz konzentriert, während der es auf verschiedenen Ebenen der Senatskarriere einen wirklichen Wettlauf bei der Suche nach Anerkennung gab, nämlich die ersten Jahre nach $60 \mathrm{v}$. Chr. Zu jener Zeit verfügte Pompeius über zwei Wohnsitze: Der eine väterliche lag im Viertel der Carinae, und sein vestibulum war sehr wahrscheinlich der Ort, von dem Cicero berichtet, er sei mit den von den Piraten eroberten Schiffsschnäbeln geschmückt gewesen; der andere nach der Errichtung des Theaters auf dem Marsfeld erbaute Wohnsitz (also vielleicht erst in der zweiten Hälfte des Jahrzehnts, wenn man annimmt, dass das Wohnhaus nach der Einweihung des Theaters im Jahre 55 v. Chr. gebaut wurde) war ein Gebäudekomplex, in den mehrere Heiligtümer eingegliedert waren. Sie waren Gottheiten geweiht, die mit der Person des Pompeius in Verbindung standen oder starke politische Resonanz hatten. Das Wohnhaus lag hinter dem Theatergebäude »wie eine Schaluppe hinter einem Schiff«, sollte Plutarch schreiben. ${ }^{6}$ Über das Wohnhaus des Crassus wissen wir leider fast Garnichts, und insbesondere wissen wir nicht, wo in der Stadt es gelegen war. ${ }^{7}$ Caesar, zumindest wenn er sich nicht auf seinem Feldzug gegen die Gallier befand und sich in der Stadt aufhielt, konnte Prestige aus seinem Amtssitz als Pontifex Maximus an der Via Sacra in der Nähe der Regia und des Hauses der Vestalinnen gewinnen, den er seit $63 \mathrm{v}$. Chr. bewohnte. In diesen Jahren blieben auch zwei Anführer der Bestrebungen der Popularen, M. Aemilius Scaurus, der Praetor des Jahres 56 v. Chr., und P. Clodius, nicht untätig. Ersterer hatte zwei Wohnstätten auf dem Palatin umgebaut oder war dabei, sie umzubauen, die in einer vornehmen Umgebung nicht weit von der Via Sacra auf einem der Hänge des Hügels lagen. ${ }^{8}$ Mit diesen Erweiterungen verfügte er über eine der größten domus von Rom, die ohne Zweifel sogar die geräumigste Wohnstatt seiner Zeit war und darüber hinaus an einem herausragenden Platz lag - vielleicht sogar in Nähe eines Erinnerungsortes, auch wenn das weniger sicher erscheint.. ${ }^{9}$ Der zweite, P. Clodius, profitierte während seines Volkstribunats im Jahre $58 \mathrm{v}$. Chr. vom Exil Ciceros und dem dazu beschlossenen Gesetz, um das Ansehen Ciceros und seines Wohnorts $\mathrm{zu}$ vernichten, indem er ihn des "Strebens nach dem Königtum" beschuldigte und seine domus nach der geltenden Praxis dem Erdboden gleichmachte. Danach wollte er, angrenzend an seine eigene domus, auf dem Palatin ein Heiligtum der Libertas errichten, das geeignet war, nach seinem Ausscheiden aus dem Amt durch die steinerne Präsenz die Verbindung von Volkstribunat und Libertas auf Dauer 
festzuhalten. Das Plädoyer ist hier sowohl in seiner strafenden Dimension wie im politischen Projekt radikal. Weniger bekannt ist die Tatsache, dass der Tribun, sobald er sich einmal mit Pompeius überworfen hatte, Letzterem damit drohte, in den Carinae ebenso vorzugehen und dort »eine zweite Portikus zu errichten, die der auf dem Palatin entspricht « ${ }^{10}$ Auf diese Weise wäre das Haus des Pompeius in den Carinae eingeebnet worden und hätte dasselbe Schicksal erlitten, wie das des Cicero auf dem Palatin. Außerdem konnte auf dem Grundstück so ein zweites öffentliches Monument errichtet werden, das in der Nähe eines anderen Hauses der Claudii lag, nämlich des Appius Claudius, Bruder des Volkstribunen. So wurde auf beiden Seiten der Via Sacra in einer vorgestellten landschaftlichen Symmetrie das Prestige der gens Claudia auf die höchste Ebene gehoben. In derselben Generation, aber diesmal in der »Partei« des Cicero, hatte T. Annius Milo, der Praetor des Jahres 55 v. Chr., beschlossen, sich zwei zentral gelegene Wohnstätten an zwei bemerkenswerten Orten zuzulegen, die eine auf dem Germalus, die andere auf dem clivus Capitolinus. ${ }^{11}$

Das ist eine schnelle Übersicht über einen begrenzten Zeitraum, mit der sich die Auswahlmöglichkeiten ermessen lassen, die zur Verfügung standen, um einem Wohnsitz hohes Prestige zu verleihen, ohne dass man dabei die treibende Kraft des Wettbewerbs vergessen darf. ${ }^{12}$ Die folgende Synthese versucht aufzuweisen, wie sich das Prestige eines Hauses abhängig von seinem Standort in der Stadt gestaltet, dann zu klären, was im Einzelnen sein Ansehen ausmacht, um abschließend einige Bemerkungen zu den Erscheinungen der Veränderung oder des Verfalls des Prestiges zu skizzieren.

\section{Verteilung im Raum und Ansehen}

4 Eine erste Reihe von Erwägungen, ${ }^{13}$ auch wenn sie auf einer eher theoretischen und synthetischen Ebene formuliert sind und man sie nicht systematisch durch konkrete Beispiele von Personen belegen kann, ergibt sich aus dem, was wir aus den für prestigeträchtig angesehenen Wahlmöglichkeiten von Wohnstätten, aus den Strategien oder Verhaltensweisen und den sie bisweilen begleitenden Äußerungen ableiten können. So wie die schriftlichen Quellen es uns darstellen, scheint die herrschende Klasse den städtischen Raum sehr wohl als polarisiert anzusehen, selbst wenn man das in Rechnung stellt oder gar zu korrigieren versucht, was man den "Cicero-Effekt « nennen könnte, d. h. die Ansicht eines homo novus mit einer sehr außergewöhnlichen Laufbahn und besonderen Ansichten. Daraus ergibt sich die Konzentration entlang bestimmter Achsen oder in berühmten oder gesuchten Zonen. Die Attraktivität ist natürlich in erster Linie durch die sichtbare Repräsentation bedingt, besonders und vor allem im Rahmen des Bürgerlebens, das als solches hauptsächlich durch die beiden Pole Forum und Marsfeld, sekundär durch deren Beziehung zum religiösen Bereich strukturiert wird. In zweiter Linie spielt die Wirkung von Geschichte und Tradition eine Rolle, die den Orten oder Gebäuden anhaftet. So hat die Nähe zu öffentlichen Gebäuden, seien sie religiös oder auch nicht, einen Einfluss auf den Wettbewerb unter Aristokraten, und besonders dann, wenn sie in persönlicher oder familiärer Verbindung mit dem Eigentümer der domus stehen (darunter auch seltene Fälle, wo man Wohnsitz und ein Heiligtum der Gens miteinander verbinden kann ${ }^{14}$ ). Ganz andere Umstände können gelegentlich in Widerspruch $\mathrm{zu}$ diesen gerade erwähnten allgemeinen Regeln stehen (die axiomatisch oder doch fast axiomatisch sind), wie etwa 
die lokale Verankerung der Gens oder die Gewährung einer Wohnstätte durch die Stadt. In diesen Fällen rührt das Ansehen der domus also nicht mehr von ihrer Lage her, sondern von anderen Elementen, die jedoch mit der Lage zusammenwirken können. So scheinen die Papirii beständig auf dem Quirinal zu wohnen, und die dem Fabricius Luscinus auf öffentliche Kosten geschenkte domus ${ }^{15}$ kann durch teilweise Übereinstimmung der Quellen im Tal zwischen Palatin und Caelius verortet werden. ${ }^{16}$ Die Identifizierung der Häuser, die über mehrere Generationen oder Jahrhunderte im Besitz derselben Familie geblieben sind, ist schwieriger. Dabei handelt es sich um einen Mangel in unseren schriftlichen Quellen, die eher dazu neigen, ihre Aufmerksamkeit auf Veränderungen im Eigentum oder die für unüblich gehaltenen oder gar skandalösen Verhaltensweisen zu konzentrieren, zum Nachteil einer Kontinuität »ohne Geschichte«.

Die bisher angeführten allgemeinen Angaben genügen, für sich genommen, nicht, um die Gesamtheit der Prestige-Parameter der Stadtwohnungen zu erhellen. Sie können im Übrigen zusammenwirken. So stellen die vom älteren Cn. Piso, dem Konsul des Jahres 7 v. Chr., gestalteten Häuser zweifellos ein beredtes Beispiel für diesen Wettlauf um das Prestige der Wohnstätten dar, und zwar besonders, wenn die von Plutarch berichtete Stichelei des Augustus gegenüber einem Piso, der ein Haus erbaute, »als ob Rom für immer überdauern müsste«, sich tatsächlich auf diese Persönlichkeit und diese domus bezieht. In diesem Fall könnte man hier sogar eine gewisse Ironie von Seiten des Autors vermuten, der als Verfasser von Kaiserbiographien das spätere Schicksal der betreffenden Residenz gut kennen musste. ${ }^{17}$ Unter dieser Annahme und im Lichte der Zeilen des Senatsbeschlusses, der das Schicksal des Gebäudes besiegelte, ging es beim Streben nach Prestige hier um die mehr oder minder unmittelbare Lage in der Nähe der Porta Fontinalis an einem Ort, der von einem Teil der Stadt aus gut sichtbar war. Dazu kam die Möglichkeit, mehrere Wohnstätten miteinander zu verbinden, wenn auch im Übergriff auf öffentliches Gelände und daher sozusagen durch Zusammenlegung mit diesem, was den Eingriff der curatores locorum publicorum iudicandorum erklärt.

Zwei Faktoren zu der Lage der Grundstücke können schließlich noch vorgebracht werden, aber das sind eher unsichere Annahmen und die Diskussion darüber bleibt weitgehend offen. Eine ziemlich gewagte Hypothese hinsichtlich der sich aus der Lage in der Stadt ergebenden Auswirkung ist die, dass der städtische Raum auch durch unbeständige oder immaterielle Besonderheiten gekennzeichnet ist, die ebenfalls Prestige bewirken. Der erste dieser Parameter, die man als Gründe für Prestige vermuten könnte, ist kürzlich erörtert und einem ohne Frage übertriebenen methodischem Zweifel unterworfen worden, und das ist die Lage an der Via triumphalis. Der traditionelle Gedanke, nach dem ein ganz und gar unveränderlicher und ritualisierter Weg für den Triumphzug und vor allem eine Umrundung des Palatin zwingend geboten war, ist in Zweifel gezogen worden. Die zuvor von Timothy Peter Wiseman und kürzlich unter anderem und ohne Anspruch auf Vollständigkeit von Mary Beard und Ida Östenberg ${ }^{18}$ formulierten Einwände schlagen eine manchmal nützliche erneute Lektüre vor, um eine Überinterpretation einiger klassischer Berichte von den Feiern der Triumphe zu vermeiden. Sie zeigen dabei eine hyperkritische Einstellung gegenüber den bruchstückhaft erhaltenen Texten und greifen häufig auf das argumentum e silentio zurück. Dennoch neigen sie nicht dazu, den Gedanken völlig in Frage zu stellen, der üblicherweise als ein Element der Lokalisierung sakraler Gebäude gilt und der besagt, dass es doch zumindest einige »feste« Abschnitte der Triumphzüge 
gibt und damit eine Reihe von Lagen, die in Hinblick auf das Prestige Träger eines starken Mehrwerts sind. Wenn es sich um private Wohnhäuser handelte, konnten diese abgesehen von der Lage an der Via sacra oder dem cliuus Capitolinus als in jeder Hinsicht bevorzugten Orten noch aus verschiedenen anderen Standorten Gewinn ziehen. Das konnte im Falle des Pompeius und seinem an das Theater gebundenen Wohnsitz ausnahmsweise das Marsfeld sein, oder sonst das Velabrum (die genaue Bedeutung des Ortsnamens bleibt umstritten), oder das Gebiet zwischen dem Palatin und dem Caelius, sei es in der Senke des künftigen Kolosseums oder auf den Hängen dieser beiden Hügel. Man hatte ohne Frage auf manchen bevorzugten Strecken - aus oft vielfältigen und sich ergänzenden Gründen - die Möglichkeit, bei jedem Triumph insbesondere durch die an der Fassade zur Schau gestellte Kriegsbeute den Sieg und die Bedeutung des Besitzers einer domus zur Geltung zu bringen oder vielmehr wieder ins Gedächtnis zu rufen. Seine Leistungen wurden so in das Gedenken der Urbs eingegliedert und wiederbelebt. Wie man in der Tat noch sehen wird, stellt die Verbindung und Einbindung der domus in den öffentlichen Raum den höchsten Grad des Prestiges dar.

7 Nicht weniger ungewiss erscheint die Möglichkeit, dass die Positionierung auf irgendwelchen privilegierten immateriellen Achsen oder in besonderen örtlichen Zusammenhängen dazu beigetragen hätte, das Prestige eines privaten oder öffentlichen Gebäudes zu begründen oder zu verstärken. Dabei könnte es sich um die Auswirkung der axialen kultischen Ausrichtung handeln, oder um die Blickrichtung (spectio) der Auguren vom auguraculum auf der Arx aus, einer Linie, die, zumindest mit Sicherheit von der augusteischen Zeit an, durch die Aufnahme archaischer oder archaisierender Traditionen die offizielle Kartographie der Stadt beeinflusst hat und seitdem die Orientierung der gedanklichen Vorstellungen bestimmt; oder um die Achse des Laufs der Sonne am dies natalis der Stadt. Auch wenn man bis jetzt noch über keine systematischen Arbeiten verfügt wie etwa die Studie über einige Bauten der Kaiserzeit durch den Architekten Piero Meogrossi, ${ }^{19}$ so gibt es da vielleicht doch noch einen Weg zu erkunden, zumindest für die Zeiträume, in denen die Bedeutung der Auspizien im Vordergrund steht. Was immer auch die Unsicherheiten dieser beiden letzten spekulativen Erwägungen sind, zweifellos ist eine Vielfalt von Maßstäben, die von der Stadt bis zu den Gebäuden reichen, notwendig, um besser zu erfassen, wie das Prestige der häuslichen Architektur zur Geltung gebracht wurde.

\section{Das Prestige eines Hauses: eine Bestandsaufnahme}

Es ist immer heikel, wenn man den Anspruch erhebt, ein Idealbild des prestigebesetzten Wohnsitzes zu entwerfen. Das läuft volens nolens auf einen Idealtyp hinaus, der eine Zusammenstellung von konstruierten Einzelheiten bleibt, die man aus verschiedenen Quellen gewonnen hat die sich zudem über einen mehr oder minder ausgedehnten Zeitraum erstrecken. Damit greift man zugegebenermaßen auf eine Methode zurück, die im 18. und 19. Jahrhundert anerkannt war. Unter der Voraussetzung, dass man sich ihrer Grenzen bewusst bleibt, könnte es sich dennoch lohnen, sie versuchsweise anzuwenden, in Anbetracht ihrer synthetischen Leistungen und sofern man so weitgehend wie möglich und in den gebührenden Grenzen auf ein aus der Regel fallendes Beispiel zurückgreift und dabei versucht, die am häufigsten betrachteten Fälle zu vermeiden. 
Abb. 1

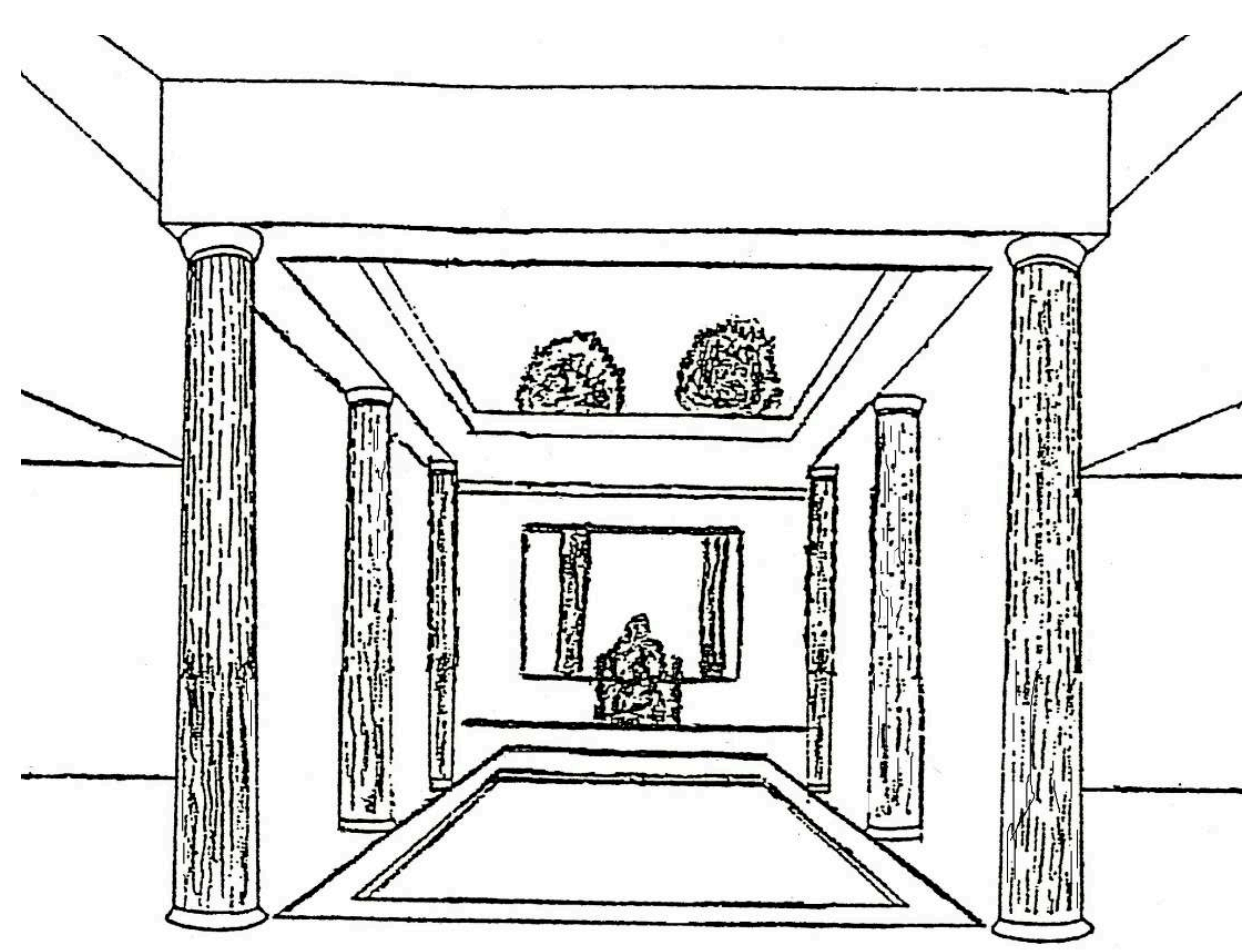

L. Licinius Crassus en Venus Palatina

9 Die Verlängerung des öffentlichen Raums und die Kontinuität mit ihm stellen mit Sicherheit den geeignetsten Parameter dar, um Prestige zum Ausdruck zu bringen. Auf materieller Ebene findet man da die symptomatische, aber ungewöhnliche Tradition in Hinblick auf die Tore, die sich zur Außenwelt hin öffnen. Plinius der Ältere erwähnt das für zwei Valerii der ältesten Epoche, Maximus und Publicola, in einer unseres Wissens völlig vereinzelten Notiz. Das bedeutet allerdings nicht zwangsläufig, dass es sich dabei um eine fehlerhafte Wiederholung oder ein bloßes Relikt von Äußerungen handelt, mit denen die gens Valeria sich selbst feierte. ${ }^{20}$ Der Eindruck der Kontinuität zwischen dem Raum der Straße und dem der domus schlägt sich als solcher im Ideal der Transparenz nieder, das man zum Beispiel auch im Rahmen der Ausübung der Funktion des Zensors formuliert vorfindet oder in dem permanenten Zugang zur Person des Tribunen oder des Rechtsberaters: ${ }^{21}$ das Wesentliche an der führenden Persönlichkeit findet sich eben dadurch zum Ausdruck gebracht. Die Aufeinanderfolge von Öffnungen oder Bögen längs eines »transparenten Ganges« (Pierre Gros), der der Folge der Umstände, dem Lauf des Tages oder des Jahres angepasst werden kann, erlaubt durch die Perspektive und die erkennbare Aussage, die sie bietet, vielfache Elemente sichtbar zu machen, manchmal durch ihre Kombination, die jedes für sich bereits Träger von Prestige sind. Die beeindruckendste Illustration des Potentials einer so in Tiefenwirkung angelegten Inszenierung wird sicherlich von dem Konsul des Jahres 95 und Zensoren des Jahres 92 v. Chr., L. Licinius Crassus, geboten, wenn man der vor einiger Zeit von Fausto Zevi vorgeschlagenen Interpretation folgt, die noch immer treffend ist, indem sie als einzige den folgenden Text des älteren Plinius in seinen spezifischen Einzelheiten vollständig erklärt (s. Abb. 1). ${ }^{22}$ 
"Schon den Redner L. Crassus, der als erster auf besagtem Palatin Säulen von ausländischem Marmor hatte, genauer gesagt aus hymettischem Marmor und nicht höher als zwölf Fuß, hatte M. Brutus in einem Streit deshalb Venus Palatina genannt.«

Die gewöhnlich vorgebrachten Erklärungen für den am Ende des Texts erwähnten Streit, die hier nicht im Einzelnen wiedergegeben werden können, reichen von der einfachen Pracht oder der Verwendung von Marmor bis zur Vermengung von Haus und Person und bleiben unklar, selbst wenn sie manchmal die Heiligtümer der Venus erwähnen und darunter das von Knidos. Natürlich ist die Bezeichnung Venus Palatina hier polemisch gemeint, aber man kann doch ohne Zweifel die Meinung vertreten, dass die visuelle Anlage, wie sie die hier abgebildete Rekonstruktion vorschlägt ${ }^{23}$ und die sich dem Auge des Besuchers oder des Passanten bot, eine sakrale Aura nahelegt wie die Venus in ihrer Tholos. Eine solche visuelle Inszenierung konnte vermutlich zum Zeitpunkt der morgendlichen Begrüßung zur Wirkung gebracht werden und grenzt damit an Illusion und Manipulation, die beide, wie bereits gezeigt, konstitutiv für Prestige sein konnten. Die visuelle Anlage bewirkte, dass das Prestige des Hauses durch die Materialien und den Schmuck sich mit dem seines Besitzers vereinte und überlagerte.

10 Auch wenn man diese angedeutete Sakralisierung letztlich nicht für erreichbar hält, lässt sich der repräsentative Bereich eines spätrepublikanischen Palais doch nicht auf die Fassade reduzieren, selbst wenn in Letzterer sich ein Großteil der Zeichen des Prestiges verdichtet. Für ihren Bereich muss man, wie zu erinnern ist, ein vielfältiges Arsenal aufzählen. Zu den bekanntesten Elementen zählen die Wandmalereien oder die an der Hausschwelle niedergelegte Kriegsbeute, ${ }^{24}$ die den Status eines "triumphalen Hauses« verleihen. Es sind im Übrigen die letzteren Elemente, die zusammen mit den im Atrium ausgestellten imagines, die nur bei großen Anlässen gezeigt und bei Trauerzügen mitgeführt werden, durch ihre Beständigkeit ganz wie die Mauern dazu beitragen können, das Prestige des "gentilizischen" Hauses der Vorfahren zu »fabrizieren«. Dazu sind noch dauerhaft oder zeitweilig aufgestellte Ehrenstatuen hinzuzuzählen oder wichtige Inschriften, und man darf auch die Liktoren eines hohen Beamten nicht vergessen, die an der Schwelle warteten, oder am Morgen die salutatores. ${ }^{25}$ Auf diese Weise trägt der vordere Teil des Hauses zum Ansehen seines Besitzers bei, nicht allein durch die Zeichen, die dort gezeigt werden, sondern auch als Ort der Zusammenkunft.

11 Man wird nach den bisherigen Erklärungen gern noch eine vierte Dimension hinzufügen, die der Zeit. Die Bestimmung des Stadthauses als Träger der Erinnerung ${ }^{26}$ und daher seine Eignung als dauerhafter Akkumulationsort von Prestige gründet sich ebenso auf seine aktive Eingliederung in mehrere kollektive Veranstaltungen, die die Menschen beeindrucken, wie auf Gegenstände, die weitere Zeichen des Prestiges des Bewohners oder seiner Vorfahren darstellen. Neben dem Triumph und der pompa funebris $^{27}$ ist dabei an die Zeremonien zu denken und an die Umzüge, um nicht Paraden zu sagen, die allesamt das politische Leben Roms markieren. Dazu gehören unter anderem die Wahlkampagnen und Wahlsiege, die Übernahme oder Aufgabe eines Amts oder das erfolgreiche Ende eines Gerichtsverfahrens. Damit ergibt sich ein außerordentliches Bild, das die domus ganz und gar in den öffentlichen Raum eingliedert und dem Gebäude und seinem Besitzer ein außergewöhnliches Prestige verleiht. Die lebhaften polemischen Angriffe, die sich gegen diese demonstrativen Umzüge richteten, etwa bei Gelegenheit der Neuordnung zum Beginn der Republik 
oder später im Zusammenhang mit Tiberius Gracchus, bestätigen, dass man es hier mit einer extremen Form zu tun hat, mit der die Bürger durch die Anwesenheit vor oder am Eingang der domus ihre Anhängerschaft zum Ausdruck bringen. Es ist sicher nicht übertrieben, bei einigen Fällen davon zu sprechen, dass sich hier kapitalisierbares Prestige manifestiert und damit die Einprägung des betreffenden Wohnhauses in die Erinnerungslandschaft der Stadt als Zielpunkt der mehr oder minder feierlichen Umzüge, und das besonders in den zentralen Stadtvierteln. So ist es durchaus statthaft anzunehmen, dass die "triumphale« Rückkehr Ciceros nach seinem Exil im Jahre 57 v. Chr. ${ }^{28}$ ein »remake« der Umzüge ist, die zunächst den Höhepunkt seines Konsulats im Jahre $63 \mathrm{v}$. Chr. nach der Ausschaltung der Catilinarier markiert haben und ebenso später das Ende seiner Zeit im Amt. Letztere hatten als Zielpunkt das Haus der Familie in den Carenae. Mit Sicherheit ist es zum Zeitpunkt seiner Rückkehr völlig ausgeschlossen, an den Rückgriff auf die domus auf dem Palatin zu denken, die Ende 62 v. Chr. von P. Clodius erworben und dann Opfer seiner erbitterten Rache geworden, also verloren und dann $58 \mathrm{v}$. Chr. abgetragen worden war. Aber es ist doch statthaft, sich Cicero als raffinierten Drahtzieher vorzustellen, der mit Genuss die Szenen vom Ende des Jahres $63 \mathrm{v}$. Chr. von neuem aufführen ließ, die für ihn einen unbestreitbaren Höhepunkt bedeutet hatten. Er konnte so durch die Inszenierung von $57 \mathrm{v}$. Chr. die Erinnerung an die langen Monate des Albtraums auslöschen, die die beiden Episoden trennen. Das Haus in den Carenae, das er an seinen Bruder Quintus abgetreten hatte, sollte so als Zielpunkt die Erinnerung an diese großartigen Umzüge und damit an diesen Teil seines am Ende des Konsulats erworbenen Ansehens bewahren. ${ }^{29}$ Wenn man dem Bericht des hauptsächlich Beteiligten folgen will, findet sich das Gebäude also zweimal in die Bühne des politischen Lebens integriert: Die Wiederherstellung der dignitas des Konsularen wird nicht allein zur Schau gestellt, sondern durch seine "triumphale« Rückkehr in seine Residenz aktualisiert und befördert, und macht vorläufig die Katastrophe des Hauses auf dem Palatin vergessen, das dann bis zu seiner Rückgabe Gegenstand aller Aufmerksamkeit wird.

Wenn man die Schwelle eines Hauses überschreitet, scheint sich das Prestige des Atriums aus seiner Übereinstimmung mit dem mos maiorum zu ergeben. Das ist zumindest der Eindruck, der sich aus der genauesten Schilderung ergibt, über die wir verfügen, der den Patrizier Aemilius Lepidus betreffend, Interrex des Jahres 52 v. Chr. ${ }^{30}$ und künftiger Triumvir. Unter den übrigen internen Zeichen von Geltung und Ansehen, von denen wir nicht genau wissen, unter welchen Bedingungen sie zugänglich und zur Schau gestellt waren, befinden sich Stoffe und Wandteppiche, Silbergerät, Statuen und Bilder, aber auch Sammlungen von sehr seltenen Gegenständen (Schmuck, Perlen oder vielleicht sogar außergewöhnliche Knochen) und kostbare Möbel. Sie gehören zu den häuslichen mirabilia ${ }^{31}$, die auch in diesem Fall ihre Entsprechungen in den Heiligtümern finden. ${ }^{32}$ Auch wenn dieser Fall einige Jahrzehnte früher liegt, kann man eine Art von Gegenprobe (bis hin zur Zurückweisung der Bezeichnung domus!) in der umfänglichen denunziatorischen Beschreibung des Wohnsitzes des Cornelius Chrysogonus auf dem Palatin finden, dessen Vorbesitzer wir trotz häufiger gegenteiliger Behauptungen nicht kennen. Der Freigelassene des Sulla wird zur besseren Diskreditierung von seinem Prozessgegner beschuldigt, eine große Zahl von Gegenständen zu besitzen (Vasen aus Korinth und Delos, einen authepsa genannten Selbstkocher, "ziseliertes Silber, Wandteppiche, Bilder, Statuen, Marmorobjekte«) und eine von Umfang und Qualität her zu verurteilende familia zu haben. Die interessanteste Bemerkung, da sie in den Bereich der artes vulgares und zur klassischen Schmährede auf die Gastmähler gehört, 
betrifft den Rückgriff auf musikalische Begleitung und offenbart in negativer Form eine noch unbekannte Form des häuslichen Prestiges. ${ }^{33}$ Zugegebenermaßen ist die Zeit zu Beginn der Bürgerkriege und der Proskriptionen durch tiefgreifende Veränderungen gekennzeichnet, die einen Teil der repräsentativen Wohnsitze in der Stadt verändert und umverteilt haben, was nicht ohne Einfluss auf die Wahrnehmung blieb, die die Römer davon hatten.

\section{Die Dynamik des Prestiges: Veränderungen und Verfall der domus, neue Hierarchien}

13 Nur wenige Texte beschreiben oder skizzieren eine ausführliche und offizielle Hierarchie innerhalb des Bereichs der städtischen Immobilien, ohne uns allerdings eine angemessene Erfassung der Kriterien zu erlauben, auf die sie sich gründet. Nach unserer Kenntnis muss man im Bereich der normativen Texte bis zur Spätantike warten, um tatsächliche juristische Festlegungen zu finden..$^{34}$ Die Erhaltung, die Veränderungen oder der Verlust von Prestige kommen in kurzen Textpassagen zum Ausdruck, die häufig nur Andeutungen enthalten. So berichtet der ältere Plinius sehr wohl von aufsehenerregenden Deklassierungen, aber er nennt kaum je den Hintergrund seines Urteils und seines Maßstabes und lässt kaum Zweifel daran, dass sein Urteil sich im Grunde aus seiner Absicht nährt, die luxuria anzuprangern. ${ }^{35}$ Das Risiko des Verfalls oder, besser gesagt, des Niedergangs, der die Gebäude bedroht, wird von Cicero ganz allgemein unterstrichen: Nach dem in den Bürgerkriegen erfolgten vielfachen Wechsel der Besitzer von Gütern, die dem Prestige dienten, ${ }^{36}$ wendet er sich in seinen berühmten Ausführungen in De officiis den städtischen Wohnhäusern zu:

»nicht aus seinem Haus soll der Hausherr Ehre gewinnen, sondern das Haus aus seinem Herrn [...]. Im übrigen bringt ein geräumiges Haus dem Herrn oft Schande ein, wenn in ihm die Einsamkeit herrscht, und am meisten, wenn es irgendwann unter einem anderen Herrn üblicherweise häufig besucht wurde. Denn es ist eine Schande, wenn von den Vorbeikommenden gesagt wird: ^Oh altes Haus, wie es von ungleichen Herren beherrscht wird .»

Der Mann aus Arpinum hat hier abgewandelt oder fast ins Gegenteil verkehrt, was er vorher über die Rolle des Hauses als suffragator bei einer Wahl der Konsuln gesagt hatte. Properz lehnt sich in einem sehr viel gefälligeren Diptychon im Zusammenhang mit dem Schicksal des Hauses der Cynthia daran an. ${ }^{37}$ Was auch immer der jeweilige Zusammenhang ist, der Wechsel des Besitzers hat so durch den Gegensatz das Prestige unterstrichen, das im kollektiven Gedächtnis an dem Haus haften blieb - so jedenfalls, wie es der Autor wahrnahm und rekonstruierte, wenn er, im Falle Ciceros, die angenommene Reaktion der Passanten in Szene setzt, die hier für ausschlaggebend angesehen wird. Man darf nicht vergessen, dass die Abhandlung zur Ethik und die aggressivsten der Philippischen Reden fast gleichzeitig entstanden sind und gegenseitige Bezüge aufweisen. Die Verweichlichung, die Profitgier und die Herrschaft der Sklaven, die in der Rede angeprangert werden, tragen dazu bei, durch die Gegensätzlichkeit die theoretischen und fast anonymen Hinweise des philosophischen Werks zu erklären. ${ }^{38}$ Indirekt erinnern sie auch an das verbleibende Prestige des Hauses des Pompeius in den Carenae. Unter den anderen Elementen, die zur Diskreditierung beitragen können, ist eine der Bemerkungen, mit der das Ansehen einer domus verbal zerstört werden kann, seine Bezeichnung als leeres Haus. ${ }^{39}$ Auf einer konkreteren Ebene ist es ohne Zweifel das Werfen von Steinen, die Steinigung, die als einzelne Handlung dem Prestige 
eines Hauses am meisten schaden könnte in einem Vorgang, der vor einiger Zeit von Paul Veyne untersucht und von ihm in die Kategorie der "Folklore« eingeordnet worden ist. ${ }^{40}$ Aber das Corpus unserer Quellen enthält in dieser Hinsicht kaum ein wirklich treffendes Beispiel, denn die wenigen Erwähnungen stehen bei Cicero und ihr Zusammenhang ist hauptsächlich rhetorisch. ${ }^{41}$

Beschränken wir uns abschließend auf die fraglichen Jahrzehnte, ohne auf eine bereits an anderer Stelle unternommene Untersuchung zurückzukommen, ${ }^{42}$ so ist für den betrachteten historischen Zeitraum zuallererst festzuhalten, dass es im Anschluss an ein nach unserer Kenntnis dramatisches Jahrhundert des Niedergangs einen spektakulären Aufstieg gab, mit der Schenkung eines Hauses durch die Stadt an Caesar im Jahre $45 \mathrm{v}$. Chr. und ab $36 \mathrm{v}$. Chr. in mehreren Etappen an Octavius-Augustus. Zweitens ist es angebracht festzustellen, dass die Residenz des Prinzeps von den ersten Jahren an bei weitem nicht mehr die eines primus inter pares war, insofern sie allein praktisch alle zuvor erwähnten Charakteristika des Ansehens einer Wohnstätte auf sich vereinigte, die Maßnahmen zur Regulierung der Stellung der anderen städtischen Wohnsitze jedoch erst in der ersten Hälfte des 2.Jahrzehnts v. Chr. durch unmissverständliche politische Signale verkündet wurden. Es handelt sich dabei einerseits um die Anbringung der Säulen des früheren Hauses des Aemilius Scaurus, das später von P. Clodius bewohnt worden war, im Theater des Marcellus, und andererseits um die aufsehenerregende Einebnung der sehr großen domus des Vedius Pollio auf dem Esquilin, um dort die Portikus der Livia zu errichten.

Um eine Zersplitterung und Verallgemeinerung der Untersuchung zu vermeiden, ${ }^{43}$ ist hier allein der städtische Wohnsitz in Betracht gezogen worden, doch es liegt auf der Hand, da es sich um die Stadt Rom handelt, dass die Gärten und die suburbanen Villen in eine Gesamtüberlegung zu der Verbindung zwischen der Art zu wohnen und dem aristokratischen Ansehen einbezogen werden müssen. Ihr Prestige zieht zumindest für einen Teil der römischen Senatoren Gewinn aus der Vernetzung zusätzlicher Wohnstätten ${ }^{44}$ die durch die häufigen Ortsveränderungen oft als solche in Szene gesetzt werden und Vorläufer des kaiserlichen Zeremoniells sind.

\section{BIBLIOGRAPHIE}

Badel, Chr. (2014): »La dignitas à Rome: entre prestige et honneur (fin de la République)«, in: Hurlet, Fr. / Rivoal, I. / Sidéra, I. (Hg.): Le Prestige. Autour des formes de la differenciation sociale (Colloques de la MAE, René-Ginouvès, 10), Paris, S. 107-118.

Beard, M. (2007): The Roman Triumph, Cambridge (Mass.).

Carandini, A. / Bruno, D. / Fraioli, F. (2010): Le case del potere nell'antica Roma, Bari und Rom.

Carandini, A. / Cappelli, R. (2000): Roma: Romolo, Remo e la fondazione della città, Mailand.

Carandini, A. / Carafa, P. (Hg.) (2012): Atlante di Roma antica: biografia e ritratti della città, Mailand.

Coarelli F. (1997): Il Campo Marzio. Dalle origini alla fine della Repubblica, Rom. 
Coarelli F. (2014): Collis: il Quirinale e il Viminale nell'Antichità, Rom.

Courrier, C. / Guilhembet, J.-P. (o. J.): »Habiter Rome (IIe s. av. J.-C.-IIe s. ap. J.-C.)«, in: Courrier C. / Guilhembet, J.-P. / Laubry, N. / Palombi, D. (Hg.): Roma, archeologia e storia urbana: l'Urbs trenta anni dopo. Convegno internazionale di studi in memoria di Ferdinando Castagnoli, Rom.

De Souza, M. / Devillers, O. (Hg.) (2019): Le Palatin, émergence de la colline du pouvoir à Rome, de la mort d'Auguste au règne de Vespasien 14-79 p.C. (Neronia, X), Bordeaux.

De Magistris, E. (2007): Paestum e Roma quadrata. Ricerche sullo spazio augurale, Salerno.

Dubouloz, J. (2011): La Propriété immobilière à Rome et en Italie, Ier - Ve siècles: organisation et transmission des praedia urbana, Rom.

Flaig, E. (2014): »Prestige et capital symbolique. Réflexions sur les funérailles aristocratiques dans la Rome républicaine«, in: Hurlet, Fr. / Rivoal, I. / Sidéra I. (Hg.): Le Prestige. Autour des formes de la differenciation sociale (Colloques de la MAE René-Ginouvès, 10), Paris, S. 197-206.

Gros, P. (2009): »Les limites d'un compromis historique: de la domus vitruvienne à la maison augustéenne du Palatin«, in: Hurlet, F. / Mineo, B. (Hg.): Le Principat d'Auguste: réalités et représentations du pouvoir autour de la »Res publica restituta«, Rennes, S. 169-185.

Guilhembet, J.-P. (2001): »Les résidences aristocratiques de Rome, du milieu du Ier siècle avant n. è. à la fin des Antonins«, Pallas, 55, S. 215-241.

Guilhembet, J.-P. (2010): „Entre Grèce et Rome, réflexions de Plutarque sur les dirigeants antiques et leur domicile: fondateurs, législateurs et refondateurs«, in: Blandenet, M. / Chillet, C. / Courrier, C. (Hg.): Figures de l'identité: naissance et destin des modèles communautaires dans le monde romain, Lyon, S. 227-245.

Guilhembet, J.-P. (2016): »Domus et monumenta: la résidence urbaine et ses pouvoirs de mémoire dans la ville de Rome (fin de la République - Haut Empire)«, in: Benoist, St. / Daguet-Gagey, A. / Hoët-Van Cauwenberghe, C. (Hg.): Une mémoire en actes, Lille.

Guilhembet, J.-P. / Royo, M. (2008): "L'aristocratie en ses quartiers (IIe siècle av. J.C. - IIe siècle apr. J.C.)«, in: Royo M. / Hubert, É. / Bérenger, A. (Hg.): »Rome des quartiers«: des vici aux rioni. Cadres institutionnels, pratiques sociales et requalifications entre Antiquité et époque moderne, Paris, S. 193-227.

Hales, S. (2003): The Roman House and Social Identity, Cambridge.

Hilbold, I. (2015): „Habiter dans des jardins«: pratiques sociales et politiques des horti résidentiels de la Ville de Rome (Ier s. av. J.-C.-Ier s. apr. J.-C.), thèse de doctorat d'histoire ancienne de l'Université de Strasbourg.

Jacotot, M. (2012): »De la philologie à la sociologie: honneur et `capital symbolique ‘ dans la Rome républicaine«, Anabases, 16, S. 189-205.

Jolivet V. (2009): »La maison romaine en Italie: planimétrie, décor et fonction des espaces«, Perspectives, 1, S. 63-68.

Lévy, J. / Lussault, M. (Hg.) (2013): Dictionnaire de la géographie et de l'espace des sociétés, Paris.

Luke, T. S. (2014): Ushering in a New Republic. Theologies of Arrival at Rome in the First Century BCE, Ann Arbor.

Madeleine, S. (2014): Le Théâtre de Pompée à Rome: restitution de l'architecture et des systèmes mécaniques, Caen. 
Meogrossi, P. (2009): »La topografia dell'urbs condita per i velaria dell'amphitheatrum «, in: Coarelli, F. (Hg.): Divus Vespasianus, Mailand, S. 116-135.

Monterosso Checa, A. (2010): Theatrum Pompei: forma y arquitectura de la génesis del modelo teatral de Roma, Madrid.

Naas, V. (2002): Le Projet encyclopédique de Pline l'Ancien, Rom.

Östenberg, I. (2010): „Circum metas fertur: an alternative Reading of the Triumphal Route«, Historia, 59, S. 303-320.

Packer, J. E. (2015): »Reconstructing Pompey's Theater«, JRA, 28 (2), S. 587-590.

Steinby, E. M. (Hg.) (1993-2000): Lexicon Topographicum Urbis Romae, 6 Bde., Rom.

Sumi, G. S. (2005): Ceremony and Power. Performing Politics in Rome between Republic and Empire, Ann Arbor.

Thomas, J.-Fr. (2014): »Le prestige politique à Rome: de l'auctoritas patricienne au prince Augustus", in: Hurlet Fr. / Rivoal, I. / Sidéra, I. (Hg.): Le Prestige. Autour des formes de la differenciation sociale (Colloques de la MAE René-Ginouvès, 10), Paris, S. 47-56.

Tucci, P. L. (2005): ")Where high Moneta leads her steps sublime Temple of Juno Moneta«, JRA, 18, S. 6-33.

Veyne, P. (1991): „Les droits de la conscience publique sur la conduite individuelle: un constat ethnologique«, in: Veyne, P.: La société romaine, Paris, S. 57-87.

Welch, K. (2006): »Domi Militiaeque: Roman Domestic Aesthetics and War Booty in the Republic«, in: Dillon, S. / Welch, K. (Hg.): Representations of War in Ancient Rome, Cambridge, S. 91-161.

Wiseman, T. P. (1994 [1987]): „Conspicui postes dignaque tecta deo. The public image of aristocratic and imperial houses in the late Republic and early Principate«, in: L'Urbs. Espace urbain et histoire (Ier s. av. J.C. - IIIe s. ap. J.C.), S. 393-413 (NDr in: ders.: Historiography and Imagination. Eight Essays on Roman Culture, Exeter, 1994, S. 98-115).

Wiseman, T. P. (2007): „Three notes on the triumphal route«, in: Leone, A. / Palombi, D. / Walker, S. (Hg.): Res bene gestae. Ricerche di storia urbana su Roma antica in onore di Eva Margareta Steinby, Rom, S. 445-449.

Wiseman, T. P. (2008): »Rethinking the Triumph«, JRA, 21, S. 389-391.

Zevi, F. (Hg.) (1991): Pompei, Neapel.

\section{NOTES}

1. Verg. Georg. 2,461-464: Si non ingentem foribus domus alta superbis / Mane salutantum totis vomis aedibus undam / Nec varios inhiant pulchra testudine postes / Illusasque auro vestes Ephyreiaque aera [...] (Übers. O. Schönberger [Reclam]). Um die Anmerkungen nicht allzusehr zu belasten, wird der sorgfältige Leser für jede domus auf den entsprechenden Artikel des Lexicon Topographicum Urbis Romae (Steinby [1993-2000]) verwiesen, um dort mehr Einzelheiten zu finden, und für die Lage auf Carandini / Carafa (2012) (manchmal nicht ohne Vorbehalt). Unter den neueren Untersuchungen auf den Spuren von Wiseman (1994 [1987]), selbst wenn sie nicht immer überzeugend sind, s. Hales (2003); Carandini / Bruno / Fraioli (2010); Bemerkungen zur Methode bei Jolivet (2009); eine historiographische Betrachtung bei Courrier / Guilhembet.

2. Zum Beispiel des Valerius Publicola erlaube ich mir auf Guilhembet (2010) zurückzuverweisen. 
3. Die Zeit, die zwischen der Rückkehr des Pompeius aus dem Osten im Jahre 61 v. Chr. und der Erbauung eines Wohnhauses außerhalb der Stadt in der Nähe seines Theaters (s. u.) zu einem uns unbekannten Datum lag und dem Jahr 3 n. Chr., als das Haus des Prinzeps auf dem Palatin nach einem Brand wiederhergestellt wurde, oder gar 14 n. Chr., als diese domus eine wichtige Rolle bei den Bestattungsfeiern des Augustus spielte.

4. Vgl. im Gegensatz dazu die sehr strikten Regeln des Munizipalgesetzes von Urso (AE 2006, 645, XIV), das ein Beispiel unter weiteren in Munizipaldokumenten erhaltenen Vorschriften darstellt. Über die Verwirrung, die häufig in Hinblick auf den Begriff des Domizils besteht, s. Dubouloz (2011), S. 166 f., vor allem Anm. 30.

5. Cic. off. 1,138f.; Vitr. 6,5, regalia in Hinblick auf die vestibula.

6. Plut. Pomp. 40,9; vgl. Coarelli (1997), S. 539-580; Monterroso Checa (2010), S. 248-261 und 390393; Madeleine (2014), S. 129-157 (vor allem S. 152-156, und dazu noch S. 8 f. die Ansicht von Filippo Coarelli, der wir uns anschließen: »die Frage müsste in ausführlicherer Weise neu aufgegriffen werden«); Packer (2015). Die weiter andauernde Diskussion um die Bestimmung der unmittelbar außerhalb am Halbkreis des Theaters liegenden Strukturen, bei denen es sich nach S. Madeleine um Fundamente des Tempels und nach A.Monterrosso Checa um eine monumentale Rampe des Zugangs zum zweiten Niveau des Gebäudes handelt, zwingen dazu, das Urteil über die möglichen Zusammenhänge zwischen dem monumentalen Komplex und dem Wohnhaus offenzulassen. Nach der neuesten Annahme des spanischen Forschers diente diese Treppe der media cavea und der summa cavea. Wenn man darin den Weg sehen will, den Pompeius von seinem nahegelegenen Wohnhaus einschlug, müsste man das in einer gewagten Vermutung als den Willen verstehen, sich nicht ohne levitas popularis seinen Weg mit der Masse der Zuschauer niederer Herkunft zu bahnen (die lex Roscia theatralis wird auf das Jahr $67 \mathrm{v}$. Chr. datiert), um vor dem neuerlichen Abstieg in die cavea die Höhe des Gebäudes und seiner Heiligtümer zu erreichen (vgl. Suet. Claud. 21,1).

7. Plut. Crass. 2,6 und 3,1-2; Cic. Cael. 9; s. auch Cic. Parad. 6,46 und Cic. Q.fr. 2,7,2; Varr. Men. 36 Cèbe; Pers. 2,36.

8. Cic. off. 1,138 .

9. Carandini / Cappelli (2000), S. 290 f. Die Cippi, deren Text auf die Geschichte von der Gründung Roms anzuspielen scheint, sind nicht eindeutig datiert und der genaue Ort ihrer Entdeckung bleibt vage; aus diesem Grunde ist die Lage des Hauses auf der Linie des ursprünglichen Pomeriums weiterhin mit Vorsicht zu betrachten.

10. Cic. har. 49: „da er (Clodius) in den Versammlungen sagte, er wolle in den Carinae eine weitere Portikus erbauen, die dem Palatin entspreche«.

11. Cic. Att. 4,3,3 (Cermalus ist eine Konjektur); Cic. Mil. 38 und 64; Cass. Dio 40,49,3-4; ILS 8393.

12. S. die interessanten Vorschläge von Flaig (2014), S. 202.

13. Zum Begriff der räumlichen Wirkung s. Lévy / Lussault (2013), S. 947-950.

14. Guilhembet / Royo (2008), S. 206-208.

15. Dazu jeweils Coarelli (2014), S. 258 f.; Guilhembet/ Royo (2008), S. 201 f. (mit anderen möglichen Beispielen) und Ps.Plac. Gloss.Lat. 5, F 36 (vgl. Val. Max. 5,3,6 und dazu Steinby (19932000), Bd. 1, S. 315 f., s.v. compitum Fabricium).

16. In anderen Fällen wird man eher Erscheinungen der Kumulation von Formen des Ansehens feststellen: die Wohnsitze der Octavii auf dem Palatin nicht weit von der Via Sacra oder der Domitii Calvini oder der Ahenobarbi auf der Velia bleiben offenbar über mehrere Generationen in der Familie; Zuweisung eines Hauses an der Via Sacra durch die Stadt an einen Scipio Nasica im 2. Jahrhundert v. Chr. (jeweils Cic. off. 1,138 und Sall. hist. frg. 2,45M, App. BC 1,64 für die Octavii; Fest. 142L, Sen. contr. 9,4,18, CIL VI 32352, 2039, 2041 und 2042d für die Domitii; Dig. 1,2,2,37 für Scipio Nasica).

17. Plut. Moral. 208A: »[...] als Piso sein Haus von den Fundamenten bis zum Dach mit großer Sorgfalt erbaute, sagte er ihm: `Du erfüllst mich mit Genugtuung, wenn du auf diese Weise baust, 
als ob Rom ewig bestehen sollte«; AE 1996, 885, Z. 106 f. Die verschiedenen Bedeutungen von supra erlauben es, ausgehend von der Formulierung supra porta Fontinalem, sich ein Bauwerk vorzustellen, das sich direkt auf der einen oder anderen Seite an das Tor selbst anlehnte, oder auch eine einfache topographische Nähe. S. dazu Tucci (2005), S. 28-30.

18. Wiseman (2007), S. 448: "Sobald wir einmal den Gedanken aufgeben, dass jeder Triumph notwendigerweise der Strecke einer archaischen lustratio des Palatin folgte [...], können wir die Möglichkeit einer langen und gewundenen Strecke durch verschiedene Teile der Stadt annehmen [...]. Der Plan der Stadt veränderte sich über die Jahrhunderte ständig und es gibt keinen Anlass $\mathrm{zu}$ bezweifeln, dass sich die Bedingungen, denen sich die Organisatoren von Triumphzügen gegenübersahen, mit ihm änderten.«; Beard (2007), S. 92-106; Wiseman (2008), S. 391; Östenberg (2010) scheint allerdings der vermutlichen Lage des Heiligtums der Fortuna recipiens keine Rechnung zu tragen (S. 308 und Anm. 20), und ebenso wenig der der oben erwähnten domus Fabricia.

19. S. dazu z. B. Meogrossi (2009), der als Anhänger einer »Archaeo-Astronomie« die Achse der spectio in Richtung auf die Albanerberge und die "Achse der Palilia« miteinander kombiniert, unter Bezugnahme auf vorangehende Beispiele (auf die Domus aurea oder die kaiserzeitlichen Gebäude der horti Luculliani). Für die privaten Bauten der spätrepublikanischen Zeit, die nur wenig oder schlecht lokalisiert sind, bleibt das Vorhaben besonders fraglich. S. auch auf anderen Grundlagen De Magistris (2007).

20. Plin. Nat. 36,112: »die großartigste Auszeichnung unter den triumphalen Häusern«, die der Autor ausdrücklich als eine Zusatzklausel des Dekrets der Zuweisung des Wohnsitzes nennt.

21. Dion. Hal. Frg.20 Pittia: "Die Römer ließen jedes Haus weit offen, und indem sie die Autorität der Zensoren bis in das Schlafzimmer ausdehnten, übertrugen sie dieser Magistratur die Sorge, alles, was sich im Inneren ereignete, zu inspizieren und zu überwachen«; zum Tribunen s. Plut. mor. 283D (QR 81); zum Rechtsberater Cic. de or. 1,200 und Cic. Tusc. 5,112.

22. Plin. nat. 36,7; Zevi (1991), S. 48 f.

23. Memoriae patris huius linearis picturae olim auctoris. Die Interpretation der anderen Texte, die die domus des Licinius Crassus betreffen, lässt an ein Atrium in Form eines korinthischen Hexastyls denken. Die Abbildung ergänzt auch in der Perspektive hypothetisch die Nesselbäume (lotus) des Hauses, die ebenfalls von sich reden gemacht haben (Plin. nat. 17,5).

24. Zu den Anfängen des Hinzukommens der Kriegsbeute in der 1. Hälfte des 2. Jahrhunderts v. Chr. s. Welch (2006).

25. Nur als Anthologie zum Thema s. Cass. Dio 46,33,2 (Statue); Cic. Aer. Al. Mil. Frg.5 (Gesetzestexte in der Eingangshalle des Clodius); Plut. Pomp. 51,4 (Liktoren in Lucca); Nonius Marcellus p. 75L (salutatio).

26. Guilhembet (2016).

27. Zuletzt dazu Flaig (2014).

28. Sumi (2005), S. 40-41, der für die Rückkehr Ciceros im Jahre 57 v. Chr. auf das Beispiel eines Triumphs verweist, aber ohne den Endpunkt des Umzugs der Volksmenge zu erwähnen; Luke (2014), S. 105-112 mit demselben Versäumnis zu dieser Episode.

29. Vgl. Cic. Att. 1,16,5 und 4,1,5; Cic. Dom. 76; Cic. Pis.7; Cic. Sest. 131; Plut. Cic. 22.

30. Asconius Mil. 43C: »nachdem sie die Tür mit aller Kraft aufgebrochen hatten, stürzten sie die Ahnenbilder um, zerstörten das kleine Hochzeitsbett seiner Ehefrau Cornelia, deren Reinheit der Sitten als vorbildmäßig galt, und zerstörten die Tuche, die man nach altem Brauch im Atrium wob«. Die Quelle des Asconius für diese Geschehnisse ist vielleicht ein offizieller Bericht.

31. Zum Begriff der mirabilia, der das Außergewöhnliche und das Wunderbare bezeichnet, s. Naas (2002), S. 243-271.

32. S. Vitr. 6,5,2 und die aufschlussreiche Skizze der häuslichen Zurschaustellung, die Cic. de or.1, 161-162 in einer Passage zur Rhetorik mit sehr konkreten Einzelheiten als Bild verwendet. Vgl. in einem völlig anderen Zusammenhang, aber zur selben Thematik des Empfangs im Wohnsitz nach 
dem mos maiorum oder zur Verhehlung des Reichtums Varr. Men. 266 Cèbe (mit dem Kommentar des Herausgebers ad loc., S. 1209, Anm. 360). Zu den Daktyliotheken und darunter der des Scaurus Plin. nat.37,11 und 9,11; zum Mobiliar im Hause des Cicero s. Cic. Att. 11,25,3 und 12,23,3 und vielleicht in Rom, aber nicht sicher Plin. nat. 13,92.

33. Cic. S.Rosc. 133-134; "um Geist und Ohren zu erfreuen, hat er so viele Leute, dass die ganze Nachbarschaft jeden Tag von Stimmen, Saiteninstrumenten oder Flöten und seinen abendlichen Gastmählern widerhallte«. S. auch Diodotos, Philosoph und Lyraspieler "nach Art der Pythagoräer«, bei Cic. Tusc. 5,113. Man wird auch an das Haus des Trimalchio im Satyricon denken.

34. So Cod. Theod. 15,2,3: »[...] summae quidem domus [...], mediocres vero et inferioris merito domus [...], ceteras vero, qui mansionem spatio angustiore sustenant [...].« Der Text bezieht sich auf die ratio dignitatis der domus; s. Dubouloz (2011), S. 566, Anm. 90.

35. Plin. nat. 36,109: das Haus des M. Aemilius Lepidus, das zum Zeitpunkt des Konsulats seines Besitzers im Jahre $78 \mathrm{v}$. Chr. das prachtvollste war, ist weniger als 35 Jahre später nur noch auf dem 100. Rang (locus).

36. Die Bedeutung ist hier eine andere als die im archäologischen und anthropologischen Sprachgebrauch übliche, wo ein »Prestigeobjekt « nicht ein Gegenstand des täglichen Nutzens ist. 37. Cic. off. 1,139; vgl. eine berühmte Wendung in Cic. Scaur. (Frg. XXII, ed. Grimal, S. 201): »depressam, caecam, iacentem domum«; Prop. 1,16,1 (»Ich für große Triumphe ehmals mich öffnende Pforte, ich unsträflicher Zucht einer Terpeia bekannt, die goldstrahlende Wagen umher an den Schwellen verherrlicht und der Gefangene mit fussfälliger Träne genetzt, ich bin jetzt verwundet vom nächtlichen Zanke der Trinker, und oft klag ich, von nicht würdigen Händen geklopft [...]« [Übers. J. H. Voss]); vgl. außerdem noch Suet. Ner. 30,2.

38. Unter anderem Cic. Phil. 2,69 (cubicula/stabula, triclinium/popina); Cic. Phil. 2,6,35 und 95; 3,10 und $30 ; 5,11$.

39. Sen. contr.2,1,1, der sich dabei sicher auf das Haus des Pompeius in den Carenae bezieht.

40. Veyne (1991), S. $76 \mathrm{f}$.

41. Cic. Att. 4,3,2; Cic. dom. 14; Cic. har. 15.

42. Guilhembet (2001), S. 219-225. Die neuen Ergebnisse der Archäologie in Hinblick auf die augusteische Zeit haben zu Diskussionen und Interpretationen geführt, die noch nicht sicher sind. Für einen ersten Ansatz s. Gros (2009) und De Souza / Devillers (2019).

43. Wobei, wie der Leser bemerkt haben wird, die lexikologischen Fragen fast ganz beiseitegelassen wurden. S. dazu zuletzt Jacotot (2012); Badel (2014); Thomas (2014).

44. $\mathrm{Zu}$ den horti s. die Dissertation von Ilse Hilbold (Hilbold [2015]), die im Juli 2015 an der Universität Strasbourg vorgelegt worden ist.

\section{INDEX}

Mots-clés : République romaine, aristocratie, représentation, prestige

Schlüsselwörter : römische Republik, Aristokratie, Repräsentation, Prestige 


\section{AUTEURS}

\section{JEAN-PIERRE GUILHEMBET}

Jean-Pierre Guilhembet ist Professor für römische Geschichte an der Université de Paris. Nähere Informationen finden Sie hier. 